Ternational Electronic Journal of Algebra

VOLUME 25 (2019) 87-103

DOI: $10.24330 /$ ieja. 504124

\title{
ANNIHILATOR CONDITIONS OF MULTIPLICATIVE REVERSE DERIVATIONS ON PRIME RINGS
}

\author{
Gurninder S. Sandhu and Deepak Kumar \\ Received: 17 March 2018; Revised: 5 August 2018; Accepted: 5 August 2018 \\ Communicated by A. Çiğdem Özcan

\begin{abstract}
Let $R$ be a ring, a mapping $F: R \rightarrow R$ together with a mapping $d: R \rightarrow R$ is called a multiplicative (generalized)-reverse derivation if $F(x y)=$ $F(y) x+y d(x)$ for all $x, y \in R$. The aim of this note is to investigate the commutativity of prime rings admitting multiplicative (generalized)-reverse derivations. Precisely, it is proved that for some nonzero element $a$ in $R$ the conditions: $a(F(x y) \pm x y)=0, a(F(x) F(y) \pm x y)=0, a(F(x y) \pm F(y) F(x))=0$, $a(F(x) F(y) \pm y x)=0, a(F(x y) \pm y x)=0$ are sufficient for the commutativity of $R$. Moreover, we describe the possible forms of generalized reverse derivations of prime rings.
\end{abstract}

Mathematics Subject Classification (2010): 16N60, 16U80, 16W25

Keywords: Prime ring, multiplicative (generalized)-reverse derivation, generalized reverse derivation, annihilator conditions

\section{Introduction}

During the time (1940-1950), when the general structure theory of rings was in progress, a significant amount of work was done by Jacobson, Herstein, Bell, Kezlan, Abu-Khuzam (see [19] and references therein) on certain polynomial constraints that force a ring to be commutative. Nowadays, there has been an ongoing interest in the investigation of polynomial constrains involving various types of derivations of rings; such constrains are usually known as differential identities. Although the notion of derivation has been existing since long back, but it took more attention of the algebraists after Posner, who established two remarkable results on derivations of prime rings (see [23, Theorem 1-2]) and hence initiated the study of relationships between the behaviour of derivations and the structure of rings. Further, the concept of derivation has been generalized in many directions for last few decades. One of these directions reports to generalized derivation, which was introduced by Brešar [7]. The study of centralizing and commuting derivations goes back to 1957, when Posner [23] proved the first notable result on centralizing (or commuting) derivations, which states that the existence of a nonzero centralizing 
derivation of a prime ring $R$ implies that $R$ is commutative. Since then, there has been a considerable interest in the study of centralizing and commuting maps of prime and semiprime rings. For this sort of work, we refer the reader to [2], [4], [5], $[6],[11],[21],[22],[24],[26]$ and references therein.

Let us turn to the brief background investigation of multiplicative derivations. The study of multiplicative derivations was initiated by Daif [10] inspired by the extraordinary work of Martindale [20] on the additivity of multiplicative isomorphisms. Later on, the complete description of these mappings was given by Goldmann and $\check{S}$ emrl [15]. In [12], Daif et al. generalized the idea of multiplicative derivation to multiplicative generalized derivation. Recently, Dhara and Ali [13] introduced the notion of multiplicative (generalized)-derivation which is a slight generalization of multiplicative generalized derivation. Moreover, Camci and Aydin [8] explored the nature of the mappings associated with multiplicative (generalized)derivations of semiprime rings. One may easily observe that the idea of multiplicative (generalized)-derivation seems more appropriate as it covers both the concepts of multiplicative derivation and multiplicative generalized derivation. During the last few years many results involving multiplicative (generalized)-derivations in prime and semiprime rings has been obtained. For a good cross section of this subject, one may see [3], [13], [16], [27], [28] and references therein.

In 1957, Herstein [17] initiated the study of reverse derivations in prime rings, where he proved that a prime ring possessing a reverse derivation is a commutative integral domain and hence reverse derivation behaves like an ordinary derivation. Amid the most recent couple of years, many authors investigated reverse derivations and extended this notion in several ways (see [1], [14], [25], [28]). Recently, Aboubakr et. al. [1] generalized the concept of reverse derivation to generalized reverse derivation and provided a study of relationship between generalized reverse derivations and generalized derivations. Inspired by this, Tiwari et. al. [28] gave the notion of multiplicative (generalized)-reverse derivation, which is a further generalization of so called $l$-generalized reverse derivation.

Throughout, $R$ is an associative ring and $Z(R)$ stands for the center of $R$. In this paper, we study the action of multiplicative (generalized)-reverse derivations on prime rings with annihilator conditions and obtain several commutativity theorems. 


\section{Preliminaries}

Definition 2.1. (i) If $R$ is a ring such that $a R b=(0)($ or $a R a=(0))$ implies either $a=0$ or $b=0$ (or $a=0$ ) for all $a, b \in R$, then $R$ is said to be a prime (or semi-prime) ring.

(ii) For any nonempty subset $S$ of $R$, the right annihilator $r_{R}(S)$ of $S$ in $R$ is the set of all $r \in R$ such that $S r=(0)$. Accordingly, the left annihilator $l_{R}(S)$ is the set of all $r \in R$ such that $r S=(0)$. The intersection of right and left annihilators of $S$ in $R$ is called annihilator of $S$ in $R$, that is

$$
A n n_{R}(S)=\{r \in R: s r=0 \text { and } r s=0 \text { for all } s \in S\}
$$

(iii) Let $H$ be a nonempty subset of $R$. Then a mapping $f: H \rightarrow R$ is said to be centralizing on $H$ if $[f(x), x] \in Z(R)$ for all $x \in H$. In particular, if $[f(x), x]=0$ for all $x \in H$, then $f$ is called commuting on $H$.

(iv) Let $R$ be a ring. An additive mapping $d: R \rightarrow R$ is said to be a derivation of $R$ if $d(x y)=d(x) y+x d(y)$ for all $x, y \in R$. For some fixed $a \in R$, a derivation $d_{a}$ is called the inner derivation of $R$ induced by $a$ if $d_{a}(x)=$ $[a, x]$ for all $x \in R$.

(v) For some fixed $a, b \in R$, a mapping $F_{a, b}: R \rightarrow R$ such that $x \mapsto a x+x b$ is called the generalized inner derivation of $R$. Immediately it follows that, if $F_{a, b}$ is generalized inner derivation, then $F_{a, b}(x y)=F_{a, b}(x) y+x d_{-b}(y)$, where $d_{-b}$ is the inner derivation of $R$ induced by $-b$. This observation of Brešar [7] gave rise to the notion of generalized derivation: An additive mapping $F: R \rightarrow R$, which is uniquely determined by a derivation $d$ of $R$ such that $F(x y)=F(x) y+x d(y)$ for all $x, y \in R$. Therefore, generalized derivations are derived from derivations in a very natural way.

(vi) A derivation $d$ which is not necessarily additive is said to be a multiplicative derivation of $R$ [10].

(vii) A mapping $F: R \rightarrow R$ (not necessarily additive) is called multiplicative (generalized)-derivation of $R$ if there exists a multiplicative derivation $d$ such that $F(x y)=F(x) y+x d(y)$ for all $x, y \in R$ [8] and [13].

(viii) If $d: R \rightarrow R$ is an additive mapping such that $d(x y)=d(y) x+y d(x)$ for all $x, y \in R$, then $R$ is called reverse derivation of $R$ [17]. Further, if we relax the assumption of additivity, then $d$ is called multiplicative reverse derivation of $R$.

(ix) An additive mapping $F: R \rightarrow R$ is said to be the $l$-generalized reverse derivation of $R$ if there exists a reverse derivation $d$ of $R$ such that $F(x y)=$ $F(y) x+y d(x)$ for all $x, y \in R[1]$. 
(x) A mapping $F: R \rightarrow R$ (not necessarily additive) together with any mapping $d: R \rightarrow R$ is said to be a multiplicative (generalized)-reverse derivation of $R$ if $F(x y)=F(y) x+y d(x)$ for all $x, y \in R[28]$.

Before going further, we construct an example to show that a reverse derivation need not to be a derivation and vice-versa.

Example 2.2. Let us consider $R=\left\{\left(\begin{array}{cccc}0 & a & b & c \\ 0 & 0 & 0 & -b \\ 0 & 0 & 0 & a \\ 0 & 0 & 0 & 0\end{array}\right): a, b, c \in \mathbb{Z}\right\}$, where $\mathbb{Z}$ is the ring of integers. We define a map $\lambda: R \rightarrow R$ such that $\lambda\left(\begin{array}{cccc}0 & a & b & c \\ 0 & 0 & 0 & -b \\ 0 & 0 & 0 & a \\ 0 & 0 & 0 & 0\end{array}\right)=$ $\left(\begin{array}{cccc}0 & 0 & -b & c \\ 0 & 0 & 0 & b \\ 0 & 0 & 0 & 0 \\ 0 & 0 & 0 & 0\end{array}\right)$. It is easy to verify that $\lambda$ is a reverse derivation on $R$ but not a derivation. Now, let $\phi: R \rightarrow R$ be a map defined by $\phi\left(\begin{array}{cccc}0 & a & b & c \\ 0 & 0 & 0 & -b \\ 0 & 0 & 0 & a \\ 0 & 0 & 0 & 0\end{array}\right)=$ $\left(\begin{array}{cccc}0 & 0 & -b & -c \\ 0 & 0 & 0 & b \\ 0 & 0 & 0 & 0 \\ 0 & 0 & 0 & 0\end{array}\right)$. This map $\phi$ is a derivation on $R$ but not a reverse derivation.

For $x, y \in R$, the symbol $[x, y]$ stands for the commutator of $x, y$ defined by $x y-y x$. In this paper, we shall use the basic commutator identities very frequently:

$$
[x, y z]=y[x, z]+[x, y] z,[x y, z]=x[y, z]+[x, z] y
$$

for all $x, y, z \in R$. Also we shall make use of the following well known result:

Lemma 2.3. [22, Lemma 3] If a prime ring $R$ contains a nonzero commutative (and hence central) right ideal, then $R$ is commutative. 


\section{Main results}

Theorem 3.1. Let $I$ be a nonzero ideal of a prime ring $R$ and $F: R \rightarrow R$ a multiplicative (generalized)-reverse derivation associated with a map $d: R \rightarrow R$. If for some $0 \neq a \in R, a(F(x y) \pm x y)=0$ for all $x, y \in I$, then $R$ is commutative.

Proof. Let us begin with

$$
a(F(x y)+x y)=0 \forall x, y \in I .
$$

Substitute $y z$ instead of $y$ in (1), we find

$$
\begin{aligned}
0 & =(a(F(y z)+y z) x)+a y z d(x)+a[x, y z] \\
& =a y z d(x)+a[x, y z]
\end{aligned}
$$

$\forall x, y, z \in I$. That is

$$
\operatorname{ayzd}(x)+a y[x, z]+a[x, y] z=0 \forall x, y, z \in I .
$$

Substituting $y$ with $a y$, in (2), we get

$$
a^{2} y z d(x)+a^{2} y[x, z]+a^{2}[x, y] z+a[x, a] y z=0 \forall x, y, z \in I .
$$

Left multiply (2) by $a$ and subtract from (3), we get $a[x, a] y z=0$ where $x, y, z \in I$. Since $R$ is a prime ring and $I$ is a nonzero ideal of $R$, we obtain

$$
a[x, a]=0 \forall x \in I .
$$

Replace $x$ by $x r$, where $r \in R$ in (4), we obtain $a[x, a] r+a x[r, a]=0$ for all $x \in I$ and $r \in R$. Application of (4) reduces it to $a x[r, a]=0$. Primeness of $R$ implies that $[r, a]=0$ as $a \neq 0$ for all $r \in R$. That means $a \in Z(R)$. But center of a prime ring does not contain a zero-divisor, so (2) yields

$$
y z d(x)+y[x, z]+[x, y] z=0 \forall x, y, z \in I .
$$

Replace $y$ by ty in (5), we obtain

$$
\operatorname{tyzd}(x)+t y[x, z]+t[x, y] z+[x, t] y z=0 \forall x, y, z, t \in I .
$$

Use of (5) gives $[x, t] y z=0$ for all $x, y, z, t \in I$. Again primeness of $R$ yields that $[I, I]=(0)$. Hence, $I$ is commutative and so by Lemma $2.3, R$ is commutative.

By doing all as above, one can prove the same conclusion for $a(F(x y)-x y)=0$ for all $x, y \in I$.

Theorem 3.2. Let $I$ be a nonzero ideal of a prime ring $R$ and $F: R \rightarrow R$ a multiplicative (generalized)-reverse derivation associated with a map $d: R \rightarrow R$. If for some $0 \neq a \in R, a(F(x) F(y) \pm x y)=0$ for all $x, y \in I$, then $R$ is commutative. 
Proof. Let us begin with

$$
a(F(x) F(y)+x y)=0 \forall x, y \in I .
$$

Substituting $y$ with $y t$ in (7), we get $a(F(x) F(t)+x t) y+a F(x) t d(y)+a(x y t-x t y)=$ 0 where $x, y, t \in I$. By hypothesis, we infer

$$
a F(x) t d(y)+a x[y, t]=0 \forall x, y, t \in I .
$$

Replace $t$ by $r t$, where $r \in R$ in (8), we obtain

$$
a F(x) r t d(y)+a x r[y, t]+a x[y, r] t=0 \forall x, y, t \in I, r \in R .
$$

Replace $x$ by $r x$ in (8), we have

$$
a F(x) r t d(y)+\operatorname{arx}[y, t]+\operatorname{axd}(r) t d(y)=0 \forall x, y, t \in I, r \in R .
$$

Subtraction of (9) from (10) gives

$$
a[r, x][y, t]+\operatorname{axd}(r) t d(y)-a x[y, r] t=0 .
$$

Substituting $x$ with ax in (11), we get

$$
a^{2}[r, x][y, t]+a[r, a] x[y, t]+a^{2} x d(r) t d(y)-a^{2} x[y, r] t=0 .
$$

Relation (11) reduces it to

$$
a[r, a] x[y, t]=0 \forall x, y, t \in I, r \in R .
$$

Since $R$ is prime, either $a[R, a]=(0)$ or $[I, I]=(0)$, that is either $a \in Z(R)$ or $R$ is commutative. Because $0 \neq a \in R$, so by (11), we find

$$
[r, x][y, t]+x d(r) t d(y)-x[y, r] t=0 \forall x, y, t \in I, r \in R .
$$

In particular, for $r=y$ in (14), we have

$$
[y, x][y, t]+x d(y) t d(y)=0 \forall x, y, t \in I .
$$

Substituting $x$ with $z x$ in (15) and then using this relation, we get $[y, z] x[y, t]=0$ for all $x, y, t \in I$, which implies $[I, I]=(0)$, implying $R$ is commutative.

By doing all as above, one can prove the same conclusion for $a(F(x) F(y)-x y)=$ 0 for all $x, y \in I$.

Theorem 3.3. Let $I$ be a nonzero ideal of a prime ring $R$ and $F: R \rightarrow R$ a multiplicative (generalized)-reverse derivation associated with a map $d: R \rightarrow R$. If for some $0 \neq a \in R, a(F(x y) \pm F(y) F(x))=0$ for all $x, y \in I$, then either $d=0$ or $R$ is commutative. 
Proof. Let us begin with

$$
a(F(x y) \pm F(y) F(x))=0 \forall x, y \in I .
$$

Replace $x$ by $z x$ in (16), we find $a(F(x y) \pm F(y) F(x)) z+a(x y d(z) \pm F(y) x d(z))=0$ for any $x, y, z \in I$. Our hypothesis yields

$$
a(x y d(z) \pm F(y) x d(z))=0 .
$$

Substitute $r y$ instead of $y$ in (17), we find

$$
a(x r y d(z) \pm F(y) r x d(z) \pm y d(r) x d(z))=0 \forall x, y, z \in I, r \in R .
$$

Replace $x$ by $r x$ in (17) and subtract it from (18), we obtain

$$
a([x, r] y d(z) \pm y d(r) x d(z))=0 .
$$

Substituting $x$ with $x r$ in (19), we get

$$
a([x, r] \operatorname{ryd}(z) \pm y d(r) \operatorname{xrd}(z))=0 .
$$

Substituting $y$ with $r y$ in (19), we obtain

$$
a([x, r] \operatorname{ryd}(z) \pm \operatorname{ryd}(r) x d(z))=0 .
$$

Combining (21) and (20), we have

$$
a([y d(r) x, r] d(z))=0 .
$$

Replace $y$ by $a y$ in (22), we find $a^{2}[y d(r) x, r] d(z)+a[a, r] y d(r) x d(z)=0$ for all $x, y, z \in I$ and $r \in R$. Application of (22) reduces it to $a[a, r] y d(r) x d(z)=0$ where $x, y, z \in I$ and $r \in R$. Primeness of $R$ implies either $a[a, R]=(0)$ or $\operatorname{Id}(I)=(0)$.

Firstly, we assume that $I d(I)=(0)$ where $z \in I$. Since $I$ is a nonzero ideal of $R$ and $R$ is a prime ring, so it implies that $d(I)=(0)$. In this case $F(x y)=F(y) x$ for any $x, y \in I$. Therefore, our hypothesis yields

$$
a F(y)(x \pm F(x))=0 \forall x, y \in I .
$$

Replace $y$ by $t y$ in (23) to obtain $a F(y) t(x \pm F(x))+a y d(t)(x \pm F(x))=0$ for all $x, y, t \in I$. Using (23), we obtain $a F(y) t(x \pm F(x))=0$. Since $R$ is a prime ring so either $a F(I)=(0)$ or $x \pm F(x)=0$ for all $x \in I$. Let us suppose that

$$
a F(y)=0 \forall y \in I .
$$

Substituting $y$ with $y r$ in (24) in order to get $a F(r) y=0$. Again, primeness of $R$ implies that $a F(R)=(0)$, which is an interesting observation. Substituting $y$ with $r y$ in (24), we get $0=a F(y) r+a y d(r)=a y d(r)$ for any $y \in I$ and $r \in R$. Since $R$ is a prime ring and $a \neq 0$, we obtain $d(R)=(0)$. 
On the other hand, if

$$
x \pm F(x)=0 \forall x \in I .
$$

On substituting $r x$ in the place of $x$ in (25), we find $r x \pm F(x) r \pm x d(r)=0$ for all $x \in I, r \in I$. That is, $[r, x]+(x \pm F(x)) r \pm x d(r)=0$ where $x \in I$ and $r \in R$. In the view of (25), it becomes

$$
[r, x] \pm x d(r)=0 \forall x \in I, r \in R .
$$

Replace $x$ by $y x$ in (26) and using it, we get $[r, y] x=0$ for all $x, y \in I$ and $r \in R$. Since $I$ is a nonzero ideal of prime ring $R$, so $R$ contains a nonzero central ideal. In the view of Lemma $2.3, R$ is commutative.

Next, we assume that $a[a, r]=0$ for all $r \in R$. Substitute $r s$ instead of $r$, we find $\operatorname{ar}[a, s]=0$ for all $r, s \in R$. Which is $a R[a, s]=(0)$, again primeness of $R$ implies $a \in Z(R)$ as $a \neq 0$. But $R$ is a prime ring, so equation (19) forces

$$
[x, r] y d(z) \pm y d(r) x d(z)=0 \forall x, y, z \in I, r \in R .
$$

Replace $y$ by $t y$ in (27), we get

$$
[x, r] \operatorname{tyd}(z) \pm \operatorname{tyd}(r) x d(z)=0 \forall x, y, z, t \in I, r \in R .
$$

Left multiply (27) by $t$ and subtract from (28), we have $[[x, r], t] y d(z)=0$. Since $R$ is a prime ring so either $[[x, r], t]=0$ for all $x, t \in I$ and $r \in R$ or $I d(I)=(0)$. Now, let us consider

$$
[[x, r], t]=0 \forall x, t \in I, r \in R .
$$

Replace $x$ by $x r$ in (29) and using it, we obtain

$$
[x, r][t, r]=0 \forall x, t \in I, r \in R .
$$

Substitute ty in place of $y$ in (30) and using it to get $[x, r] t[y, r]=0$ for all $x, y, t \in I$ and $r \in R$. Again, primeness of $R$ implies that $R$ contains a nonzero central ideal and hence $R$ is commutative with the aid of Lemma 2.3.

Since $R$ is prime ring, the latter case $I d(I)=(0)$ implies that $d(I)=(0)$. Therefore, in this case $F(x y)=F(y) x$ for all $x, y \in I$. So our hypothesis yields that $F(x)(y \pm F(y))=0$ for any $x, y \in I$, by following the same procedure as in the first case we get $d(R)=0$.

In this sequel, it is natural to consider the following theorem:

Theorem 3.4. Let $I$ be a nonzero ideal of a prime ring $R$ and $F: R \rightarrow R$ a multiplicative (generalized)-reverse derivation associated with a map $d: R \rightarrow R$. If 
for some $0 \neq a \in R, a(F(x y) \pm F(x) F(y))=0$ for all $x, y \in I$, then either $d=0$ or $R$ is commutative.

In order to prove this claim, we need to develop some more results. Hence, we shall give the proof in the end.

Theorem 3.5. Let $I$ be a nonzero ideal of a prime ring $R$ and $F: R \rightarrow R$ a multiplicative (generalized)-reverse derivation associated with a map $d: R \rightarrow R$. If for some $0 \neq a \in R, a(F(x) F(y) \pm y x)=0$ for all $x, y \in I$, then $R$ is commutative.

Proof. Let us begin with

$$
a(F(x) F(y)+y x)=0 \forall x, y \in I .
$$

Replace $y$ by $z y$ in (31), we get $a(F(x) F(y)+y x) z+a(F(x) y d(z)+[z, y x])=0$ for all $x, y, z \in I$. By hypothesis, we find

$$
a(F(x) y d(z)+y[z, x]+[z, y] x)=0 \forall x, y, z \in I .
$$

Substitute $z x$ for $x$ in (32), we obtain

$$
a(F(x) z y d(z)+x d(z) y d(z)+y z[z, x]+[z, y] z x)=0 .
$$

Replace $y$ by $y z$ in (32), we find

$$
a(F(x) y z d(z)+y z[z, x]+[z, y] y x)=0 .
$$

Subtract (34) from (33) in order to find

$$
a(F(x)[z, y] d(z)+x d(z) y d(z))=0 \forall x, y, z \in I .
$$

Replace $x$ by $z x$ in (35), we obtain

$$
a(F(x) z[z, y] d(z)+x d(z)[z, y] d(z)+z x d(z) y d(z))=0 .
$$

Substituting $y$ with $z y$ in (35), we have

$$
a(F(x) z[z, y] d(z)+x d(z) z y d(z))=0 .
$$

Subtract (36) from (37), we get

$$
a([x d(z), z] y d(z)+x d(z)[y, z] d(z))=0 \forall x, y, z \in I .
$$

Substitute $a x$ instead of $x$ in (38), we obtain

$$
a[\operatorname{axd}(z), z] y d(z)+a^{2} x d(z)[y, z] d(z)=0 .
$$

Left multiply (38) by $a$ and subtract it from (39), we find $[a, z] x d(z) y d(z)=0$ for all $x, y, z \in I$. Therefore, primeness of $R$ forces either $[a, I]=(0)$ or $\operatorname{Id}(I)=(0)$. That means either $a \in Z(R)$ or $d(I)=(0)$. 
Firstly, if $d(I)=(0)$, then (32) becomes

$$
a y[z, x]+a[z, y] x=0 \forall x, y, z \in I .
$$

Putting $y=a y$ in relation (40) in order to find

$$
a^{2} y[z, x]+a^{2}[z, y] x+a[z, a] y x=0 \forall x, y, z \in I .
$$

Using (40) in (41), we obtain $a[z, a] y x=0$. Since $R$ is prime ring and $I$ a nonzero ideal of $R$, we get $a[a, z]=0$ for all $z \in I$. Replacing $z$ by $z r$, where $r \in R$, we get $a I[a, r]=(0)$. It implies that $a \in Z(R)$. But center of a prime ring does not contain zero-divisor, hence (40) implies

$$
y[z, x]+[z, y] x=0 .
$$

Replace $x$ by $x r$ in (42), where $r \in R$ and using it, we obtain $y x[z, r]=0$ for all $x, y, z \in I$ and $r \in R$. Primeness of $R$ implies $I \subseteq Z(R)$. By Lemma 2.3, $R$ is commutative.

On the other hand, if $a \in Z(R)$ and $R$ is a prime ring so (32) yields

$$
F(x) y d(z)+y[z, x]+[z, y] x=0 \forall x, y, z \in I .
$$

Replace $x$ by $z x$ in (43) and we have

$$
F(x) z y d(z)+y z[z, x]+[z, y] x z+x d(z) y d(z)=0 .
$$

Substitute $z y$ for $y$ in (43) and subtract from (44) to obtain

$$
[y, z][z, x]+[[z, y], z] x+x d(z) y d(z)=0 .
$$

Replace $x$ by $z x$ in (45) and we find

$$
[y, z] z[z, x]+[[z, y], z] z x+z x d(z) z y d(z)=0 .
$$

Left multiply (45) by $z$ and subtract it from (46) in order to obtain $[[y, z], z][z, x]+$ $[[[z, y], z], z] x=0$. By replacing $x$ with $x t$ in the last expression and using it, we obtain $[[y, z], z] x[z, t]=0$. In particular, we get $[[y, z], z] I[[y, z], z]=(0)$. It implies that $[[y, z], z]=0$ for all $y, z \in I$. From this it is easy to conclude that $I$ is commutative and so is $R$.

By doing all as above, one can prove the same conclusion for $a(F(x) F(y)-y x)=$ 0 for all $x, y \in I$.

Theorem 3.6. Let $I$ be a nonzero ideal of a prime ring $R$ and $F: R \rightarrow R a$ multiplicative (generalized)-reverse derivation associated with a map $d: R \rightarrow R$. If for some $0 \neq a \in R, a(F(x y) \pm y x)=0$ for all $x, y \in I$, then $R$ is commutative. 
Proof. Let us begin with

$$
a(F(x y)+y x)=0 \forall x, y \in I .
$$

Substituting $x$ with $z x$ in (47), we get $a(F(x y)+y x) z+a(x y d(z)+y[z, x])=0$ for all $x, y, z \in I$. By hypothesis, we find

$$
a(x y d(z)+y[z, x])=0 .
$$

Replace $x$ by $a x$ in (48), we obtain

$$
a^{2} x y d(z)+a y a[z, x]+a y[z, a] x=0 .
$$

Left multiply (48) by $a$, we find

$$
a^{2} x y d(z)+a^{2} y[z, x]=0 .
$$

Subtract (50) from (49) and we infer

$$
a([y, a][z, x]+y[z, a] x)=0 .
$$

Replace $z$ by $z x$ in (51), we get

$$
a\left([y, a][z, x] x+y[z, a] x^{2}+y z[x, a] x\right)=0 .
$$

Combining (51) and (52), we have

$$
a(y z[x, a] x)=0 .
$$

Putting $y=r y$ in (53), where $r \in R$, we obtain $a R y z[x, a] x=(0)$. Since $a \neq 0$ and $R$ is a prime ring, we must have $y z[x, a] x=0$ for all $x, y, z \in I$. Again primeness of $R$ implying $[x, a] x=0$ for all $x \in I$. Linearizing w.r.t. $x$, we get

$$
[x, a] y+[y, a] x=0 \forall x, y \in I .
$$

Substituting $x$ with $x r$ in (54), we find

$$
[x, a] r y+x[r, a] y+[y, a] x r=0 \forall x, y \in I, r \in R .
$$

Right multiply (55) by $r$ and subtract from (54), we get

$$
[x, a][r, y]+x[r, a] y=0 .
$$

Replace $y$ by $y z$ in (56), we obtain

$$
[x, a][r, y] z+[x, a] y[r, z]+x[r, a] y z=0 .
$$

Relation (56) reduces it to

$$
[x, a] y[r, z]=0 \forall x, y, z \in I, r \in R .
$$


Since $R$ is a prime ring so either $[I, a]=(0)$ or $[R, I]=(0)$. That means, either $a \in Z(R)$ or $R$ contains a nonzero central ideal. It is clear from Lemma 2.3 that if $R$ contains a nonzero central ideal then $R$ is commutative. On the other hand, let us assume that $a \in Z(R)$ and $R$ is a prime ring. Therefore, relation (48) forces

$$
x y d(z)+y[z, x]=0 \forall x, y, z \in I .
$$

Substitute $r x$ in place of $x$ in (59), we get

$$
\operatorname{rxyd}(z)+y r[z, x]+y[z, r] x=0 \forall x, y, z \in I, r \in R .
$$

Left multiply (59) by $r$ and subtract from (60), we find

$$
[y, r][z, x]+y[z, r] x=0 .
$$

Replace $x$ by $x r$ in (61) and using it to obtain $[y, r] x[z, r]=0$ for all $x, y, z \in I$ and $r \in R$. Again, primeness of $R$ implies that $R$ contains a nonzero central ideal. Hence, $R$ is commutative.

By doing all as above, one can prove the same conclusion for $a(F(x y)-y x)=0$ for all $x, y \in I$.

In the direction of Herstein [17], we obtain the following results on multiplicative reverse derivations:

Corollary 3.7. Let $I$ be a nonzero ideal of a prime ring $R$ and $F: R \rightarrow R a$ multiplicative (generalized)-reverse derivation together with a map $d: R \rightarrow R$. If any one of the following identities hold true

1. $a(F(x y) \pm x y)=0$

2. $a(F(x y) \pm y x)=0$

3. $a(F(x) F(y) \pm x y)=0$

4. $a(F(x) F(y) \pm y x)=0$

for all $x, y \in I$, then $R$ is a commutative integral domain and $F$ is a multiplicative (generalized)-derivation of $R$.

Remark 3.8. It is easy to see that the above conditions are not necessary for the commutativity of a prime ring. Let $F: Z_{2}[x] \rightarrow Z_{2}[x]$ be the ordinary differential operator on the polynomial ring $Z_{2}[x]$. Let us consider $F$ as a multiplicative (generalized)-reverse derivation associated with a map $d=F$ and we have $F(f g)=F(g) f+g d(f)$ for all $f, g \in Z_{2}[x]$. Now, note that the above conditions (1-4) are not always true but $Z_{2}[x]$ is the commutative integral domain. 
Remark 3.9. Let $F$ be a multiplicative (generalized)-reverse derivation of ring $R$ together with a map $f$ and $\lambda: R^{*} \rightarrow R$ be a multiplicative left centralizer, where $R^{*}$ denotes the opposite ring of $R$. Then the mapping $G=F \pm \lambda$ is a multiplicative (generalized)-reverse derivation associated with map $f$.

Example 3.10. $R=\left\{\left(\begin{array}{ccc}0 & m & n \\ 0 & 0 & p \\ 0 & 0 & 0\end{array}\right): m, n, p \in Z\right\}$, where $Z$ is the ring of integers. Define a mapping $d: R \rightarrow R$ such that $d\left(\begin{array}{ccc}0 & m & n \\ 0 & 0 & p \\ 0 & 0 & 0\end{array}\right)=\left(\begin{array}{ccc}0 & m & n p \\ 0 & 0 & -p \\ 0 & 0 & 0\end{array}\right)$. Clearly, $d$ is a multiplicative reverse derivation. Let $F=d$ be the multiplicative (generalized)-reverse derivation associated with $d$. It is easy to check that $F$ satisfies all the polynomial constraints: $a(F(x y) \pm x y)=0, a(F(x y) \pm y x)=0$, $a(F(x) F(y) \pm x y)=0$ and $a(F(x) F(y) \pm y x)=0$ but $R$ is not commutative. Hence the imposed restriction of primeness on $R$ is not superfluous.

Next we construct the following example, which is showing that some of our theorems can not be extended to semiprime rings.

Example 3.11. Consider a semiprime ring $S$ and a domain $D$. Let $R=S \times D$ and $\phi$ be a multiplicative reverse derivation of $S$ so clearly $\phi(0)=0$. We define $F: R \rightarrow R$ and $d: R \rightarrow R$ respectively as: $F\left(x_{1}, x_{2}\right)=\left(\phi\left(x_{1}\right), x_{2}\right)$ and $d\left(x_{1}, x_{2}\right)=$ $\left(\phi\left(x_{1}\right), 0\right)$. Then $F$ is a multiplicative (generalized)-reverse derivation associated with a map $d$ on $R$ and clearly $F$ is not a multiplicative (generalized)-derivation. The set $I=\{0\} \times D$ is an ideal of $R$. Now, it is easy to see that $a(F(x y) \pm x y)=0$ and $a(F(x) F(y) \pm x y)=0$ for all $x, y \in I$; but $R$ is not a commutative ring.

If $R$ is a prime ring, then $Q_{r}(R)$ and $Q_{s}(R)$ denotes the right and symmetric Martindale ring of quotients respectively. It is a well known fact that centers of $Q_{r}(R)$ and $Q_{s}(R)$ coincide and denoted by $C$, which is called the extended centroid of $R$. The symbol $R C=R_{C}$ denotes the central closure of $R$, which is again a prime ring, hence we may construct $Q_{r}\left(R_{C}\right)$. In this section, we explore the structure of the mapping $d$ which is associated with multiplicative (generalized)-reverse derivation $F$ of $R$. Precisely, we show that in the definition of a multiplicative (generalized)reverse derivation of a prime ring $R, d$ needs not necessarily be a reverse derivation of $R$. 
Proposition 3.12. Let $R$ be a prime ring and $F: R \rightarrow R$ the multiplicative (generalized)-reverse derivation of $R$ associated with a mapping $d: R \rightarrow R$. Then either $R$ is commutative or $d$ is the multiplicative left centralizer.

Proof. For any $x, y, z \in R$, we find $F(x z y)=F(z y) x+z y d(x)$. That is,

$$
F(x z y)=F(y) z x+y d(z) x+z y d(x)
$$

On the other hand, we obtain

$$
F(x z y)=F(y) x z+y d(x z)
$$

Subtract (63) from (62), we obtain

$$
F(y)[z, x]+y d(z) x+z y d(x)-y d(x z)=0
$$

Replace $x$ by $x z$ in (64), we get

$$
F(y)[z, x] z+y d(z) x z+z y d(x z)-y d\left(x z^{2}\right)=0
$$

Right multiply (64) by $z$ and subtract it from (65), we obtain

$$
z y d(x z)-y d\left(x z^{2}\right)-z y d(x) z+y d(x z) z=0
$$

Substitute ty instead of $y$ in (66), we find

$$
z t y d(x z)-t y d\left(x z^{2}\right)-z t y d(x) z+t y d(x z) z=0
$$

Left multiply (66) by $t$ and subtract it from (67), we get $[z, t] y(d(x z)-d(x) z)=0$ for all $x, y, z, t \in R$. That is, $[z, t] R(d(x z)-d(x) z)=(0)$ for all $x, z, t \in R$. Hence, primeness of $R$ ensures our conclusion.

Now, we are in the position to prove Theorem 3.4

Proof of Theorem 3.4. Let us assume that $R$ is noncommutative. By hypothesis, $a(F(x y) \pm F(x) F(y))=0$ for all $x, y \in I$. That is,

$$
(F(y) x+y d(x) \pm F(x) F(y))=0
$$

Replace $y$ by $z y$ in (68), we find

$$
a(F(y) z x+y d(z) x+z y d(x) \pm F(x) F(y) z \pm F(x) y d(z))=0
$$

Right multiply (68) by $z$ and subtract (69) from it, we obtain

$$
a(F(y)[x, z]+[y d(x), z]-y d(z) x \pm F(x) y d(z))=0
$$

Substituting $z$ with $z x$ in (70) and applying Proposition 3.12, we get

$$
a\left(F(y)[x, z] x+[y d(x), z] x+z[y d(x), x]-y d(z) x^{2} \pm F(x) y d(z) x\right)=0
$$


Using (70) in (71), we are left with $z[y d(x), x]=0$ for all $x, y, z \in I$. For any $t \in I$, replace $y$ by $t y$, we find $z t[y d(x), x]+z[t, x] y d(x)=0$. It yields $z[t, x] y d(x)=0$ for all $x, y \in I$. Since $R$ is a prime ring so either $I$ is commutative or $d(I)=(0)$. In the view of our assumption, $d$ is zero on $I$ and hence on $R$.

Corollary 3.13. Let $R$ be a noncommutative prime ring and $F: R \rightarrow R$ be a mapping of $R$ satisfying $F(x+y)=F(x)+F(y)$ and $F(x y)=F(y) x+y d(x)$ for all $x, y \in R$ and a mapping $d$ of $R$. Then $d$ is a left centralizer of $R$.

Theorem 3.14. Every generalized reverse derivation $F$ of a noncommutative prime ring $R$ is of the form $F(x)=-x q$ for all $x \in R$ and fixed $q \in Q_{r}\left(R_{C}\right)$.

Proof. Since $F$ is a generalized reverse derivation of $R$ associated with left centralizer $d$ of $R$. In the light of Hvala [18], these exist a $q \in Q_{r}\left(R_{C}\right)$ such that $d(x)=q x$ for all $x \in R$. From Eq. (64), we have $F(y)[z, x]+y q z x+z y q x-y q x z=0$ for all $x, y, z \in R$. That gives, $(F(y)+y q)[z, x]+z y q x=0$. Replacing $x$ by $x r$ in the last relation, we get $(F(y)+y q) R[r, y]=(0)$. Since $R$ is prime, we get $F(y)=-y q$ for all $y \in R$.

Corollary 3.15. Let $F: R \rightarrow R$ be a generalized reverse derivation of a noncommutative prime ring $R$. If $F$ is commuting on $R$, then there exists some $q \in C$ such that $F(x)=-q x$ for all $x \in R$.

Proof. By hypothesis, $[F(x), x]=0$ for all $x \in R$. In the view of theorem 3.14, we have $-[x q, x]=0$ for some $q \in Q_{r}\left(R_{C}\right)$. That is, $x[q, x]=0$ for all $x \in R$. Hence, by Theorem (I) of [9], we are done.

Acknowledgement. We are thankful to Prof. Basudeb Dhara for his helpful suggestions. We would also like to express our sincere gratitude to the anonymous referee for his/her constructive comments that definitely improve the readability of the paper.

\section{References}

[1] A. Aboubakr and S. González, Reverse generalized derivations of semiprime rings, Sib. Math. J., 56(2) (2015), 199-205.

[2] A. Ali, D. Kumar and P. Miyan, On generalized derivations and commutativity of prime and semiprime rings, Hacet. J. Math. Stat., 40(3) (2011), 367-374.

[3] S. Ali, B. Dhara, N. A. Dar and A. N. Khan, On Lie ideals with multiplicative (generalized)-derivations in prime and semiprime rings, Beitr. Algebra Geom., 56(1) (2015), 325-337. 
[4] M. Ashraf, A. Ali and S. Ali, Some commutativity theorems for rings with generalized derivations, Southeast Asian Bull. Math., 31(3) (2007), 415-421.

[5] M. Ashraf and N. Rehman, On derivation and commutativity in prime rings, East-West J. Math., 3(1) (2001), 87-91.

[6] H. E. Bell and W. S. Martindale, III, Centralizing mappings of semiprime rings, Canad. Math. Bull., 30(1) (1987), 92-101.

[7] M. Brešar, On the distance of composition of two derivations to the generalized derivation, Glasgow Math. J., 33(1) (1991), 89-93.

[8] D. K. Camci and N. Aydin, On multiplicative (generalized)-derivations in semiprime rings, Commun. Fac. Sci. Univ. Ank. Sér. A1 Math. Stat., 66(1) (2017), 153-164.

[9] C. M. Chang and Y. C. Lin, Derivations on one-sided ideals of prime rings, Tamsui Oxf. J. Math. Sci., 17(2) (2001), 139-145.

[10] M. N. Daif, When is a multiplicative derivation additive?, Internat. J. Math. Math. Sci., 14(3) (1991), 615-618.

[11] M. N. Daif and H. E. Bell, Remarks on derivations on semiprime rings, Internat. J. Math. Math. Sci., 15(1) (1992), 205-206.

[12] M. N. Daif and M. S. Tammam-El-Sayiad, Multiplicative generalized derivations which are additive, East-West J. Math., 9(1) (2007), 31-37.

[13] B. Dhara and S. Ali, On multiplicative (generalized)-derivations in prime and semiprime rings, Aequationes Math., 86(1-2) (2013), 65-79.

[14] A. K. Faraj, On generalized $(\theta, \phi)$-reverse derivations of prime rings, Iraqi J. Sci., 52(2) (2011), 218-224.

[15] H. Goldmann and P. Šemrl, Multiplicative derivations on $C(X)$, Monatsh. Math., 121(3) (1996), 189-197.

[16] I. Gusic, A note on generalized derivations of prime rings, Glas. Mat. Ser. III, 40(60) (2005), 47-49.

[17] I. N. Herstein, Jordan derivations of prime rings, Proc. Amer. Math. Soc., 8 (1957), 1104-1110.

[18] B. Hvala, Generalized derivations in rings, Comm. Algebra, 26(4) (1998), 11471166.

[19] J. Pinter-Lucke, Commutativity conditions for rings: 1950-2005, Expo. Math., 25(2) (2007), 165-174.

[20] W. S. Martindale, III, When are multiplicative mappings additive?, Proc. Amer. Math. Soc., 21 (1969), 695-698. 
[21] H. Marubayashi, M. Ashraf, N. Rehman and S. Ali, On generalized ( $\alpha, \beta)$ derivations in prime rings, Algebra Colloq., 17(Spec. 1) (2010), 865-874.

[22] J. H. Mayne, Centralizing mappings of prime rings, Canad. Math. Bull., 27(1) (1984), 122-126.

[23] E. C. Posner, Derivations in prime rings, Proc. Amer. Math. Soc., 8 (1957), 1093-1100.

[24] N. Rehman, On commutativity of rings with generalized derivations, Math. J. Okayama Univ., 44 (2002), 43-49.

[25] M. Samman and N. Alyamani, Derivations and reverse derivations in semiprime rings, Int. Math. Forum, 2(39) (2007), 1895-1902.

[26] G. S. Sandhu and D. Kumar, A note on derivations and Jordan ideals of prime rings, AIMS Math., 2(4) (2017), 580-585.

[27] G. S. Sandhu and D. Kumar, Derivable mappings and commutativity of associative rings, Italian J. Pure Appl. Math., 40 (2018), 376-393.

[28] S. K. Tiwari, R. K. Sharma and B. Dhara, Some theorems of commutativity on semiprime rings with mappings, Southeast Asian Bull. Math., 42(2) (2018), 279-292.

\author{
Gurninder S. Sandhu (Corresponding Author) \\ Department of Mathematics \\ Punjabi University \\ 147002 Patiala, India \\ and \\ Multani Mal Modi College \\ 147001 Patiala, India. \\ e-mail: gurninder_rs@pbi.ac.in
}

\title{
Deepak Kumar
}

Department of Mathematics

Punjabi University

147002 Patiala, India

e-mail: deep_math1@yahoo.com 